\title{
The Gas Liquid Partition Coefficients of Eleven Normal, Branched and Cyclic Alkanes in Sixty Nine Common Organic Liquids II: The Effect of Solvent Structure
}

\author{
Won Jo Cheong \\ Department of Chemistrv and Institute for Basic Research, Inha Cniversin, Incheon $402-751$. Korea \\ Received March 28, 2003
}

\begin{abstract}
The effect of solvent structure on the slope in the plot of $\ln K^{r}$ us. solute carbon number was examined. It was found that the free energy of methylene group transfer from the gas plase into a solvent was always negative and that the absolute magnitude of interaction free energy between the methylene group and the solvent was always larger than the absolute magnitude of cavity formation free energy of the methylene group in the solvent. Thus, the slope in the plot of $\ln K$ is. solute carbon number was always positive and its value decreases with increase of solvent polarity since the cavity formation energy of the $\mathrm{CH}_{2}$ unit increases with increase of solvent polarity while the dispersive interaction energy of the $\mathrm{CH}_{2}$ unit is virtually invariant. We also examined the effect of sequential addition of $\mathrm{CH}_{2}$ unit to a solvent molecule upon $\ln K$ for three homologous series of solvents: $n$-alkanes, $n$-alcohols. and $n$-nitriles. Characteristic trends in the plots of $\ln K v$. solvent carbon number were observed for individual solvent groups. A decrease of $\ln K$ with solvent carbon number was observed for $n$-alkanes. An abrupt increase in $\ln K$ followed by levelling off was observed for $n$-alcohols while a final slight decrease in $\ln K$ after an abrupt increase followed by rapid levelling off was noted for $n$-nitriles. All of theses phenomena were found related to variation in cavity formation energy. It was clearly shown that a structural change of a polar solvent by sequential addition of $\mathrm{CH}_{2}$ units causes an abrupt polarity decrease initially. then gradual levelling off. and finally. conversion to a virtually nonpolar solvent if enough $\mathrm{CH}_{2}$ units are added.
\end{abstract}

Key Words : Partition coefficients. Alkane solutes. Solvent structure. Cavity formation. Dispersive interaction

\section{Introduction}

The gas liquid partition coefficients and activity coefficients of alkanes used to be the data of importance in many fields of chemistry. including chromatography ${ }^{1-5}$ plysical organic chemistry ${ }^{6.7}$ and chemical engineeering. ${ }^{8-11}$ Partition coefficient data are directed to related to the process of solution. Various models of solution process have been studied. Conder and Young have summarized many of these studies and models. ${ }^{\text {l }}$ Alkanes are the least complex probe solutes because the only possible attractive forces between an alkane and the solvent are dispersive interactions.

In the previous paper." we critically examined the effect of solute structure on the logarithunic gas/liquid partition coefficients of normal. branched. and cyclic alkanes in 69 organic solvents. and determined the effective carbon numbers $(\mathrm{ECN})$ for the branched and cyclic alkanes and figured out the physicochemical meaning of ECN.

In this paper. the effects of solvent structure on the logarithmic partition coefficients of normal. branched. and cyclic alkanes will be discussed.

\section{Theory}

The gas liquid partition coefficients used in this work are defined by equation 1

$$
K=[\text { solute }]_{\text {ıqud }} /[\text { solute }]_{\text {gas }}
$$

The free energy of solution(the free energy change owing to the solute transfer from the gas phase to the liquid phase) of the solute corresponds to the process:

$$
\text { solute (gas, I M) } \rightleftarrows \text { solution (liquid, I M) }
$$

is given by:

$$
\Delta G^{\circ}=-\mathrm{RT} \ln K
$$

If the gas phase is regarded ideal. then the activity coefficient (Raoult's law based and mole fraction based) is computed from the following equation:

$$
\Upsilon^{*}=\frac{d_{1} R T}{K p^{\circ} M_{1}}
$$

where $d$ is the density of the solvent. $K$. the gas liquid partition coefficient. $p^{\circ}$. the vapor pressure of the pure solute. and $M$. the molecular weight of the solvent.

We define a series of homologous solutes as a group that is composed of an initial solute and solutes with sequentially added $\mathrm{CH}_{2}$ groups. A group composed of pentane, hexane. heptane. octane nonane and a group composed of methanol. ethanol. n-propanol. $n$-butanol. n-pentanol. are good examples of homologous series. The solute is composed of the head group and the alkyl chain group. The free energy of transfer of a solute can be divided into two terms. the contribution of the head group and the contribution of the alkyl chain group. The contribution of the alkyl group is composed of the individual contributions of the $\mathrm{CH}_{2}$ units and the terminal $\mathrm{CH}_{2}$ unit. The difference between the $\mathrm{CH}_{2}$ and $\mathrm{CH}_{3}$ units is. 
in general negligible and the following relationship is well established for a homologous series:

$$
\Delta G^{\circ}=\Delta G_{a}^{\circ}+n \Delta G_{b}^{\circ}
$$

where $\Delta G^{\circ}$ is is the head group contribution. n. the number of $\mathrm{CH}_{2}$ or $\mathrm{CH}_{3}$ groups, and $\Delta G_{b}^{\circ}$. the $\mathrm{CH}_{2}$ (or $\mathrm{CH}_{3}$ ) group contribution.

Equation 5 can be easily converted to Equation 6

$$
\ln K=a+n b
$$

where $a=-\Delta G_{u}^{\circ} /(\mathrm{RT})$, and $b=-\Delta G_{b}^{\circ} /(\mathrm{RT})$

Based on the accuracy of the Kovats retention index scheme ${ }^{13}$ and many models of the solution process. ${ }^{1420}$ these plots $(\ln K w . n)$ are generally quite linear. thus. correlation coefficients are greater than 0.9995 , and the average deviations are less than $0.05 \mathrm{in} \ln K$ units.

The methylene group $\left(\mathrm{CH}_{2}\right)$ contribution to the free energy of solute transfer from the gas phase to the solvent can be divided into two components: the cavity formation free energy and the interaction free energy of $\mathrm{CH}_{2}$ with the solvent. The cavity formation energy is required to make a cavity for the $\mathrm{CH}_{2}$ unit in the solvent. thus the sign of the cavity formation free energy is always positive while the sign of the interaction free energy is alway's negative.

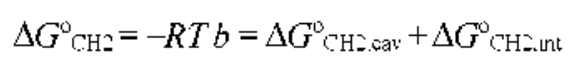

$$
(+) \quad(-)
$$

where $\Delta G^{\circ} \mathrm{CH}$, cav is the cavity formation free energy of methylene group. and $\Delta G^{\circ} \mathrm{CH} \nu$,int is the interaction free energy of methylene group. The interaction of $\mathrm{CH}_{2}$ with the solvent is almost dispersive and $\Delta G^{\circ} \mathrm{CH}$, int is virtually invariant over the whole range of solvents. while $\Delta G^{\circ} \mathrm{CH}$.cay is strongly dependent on solvent polarity. Since the cavity formation energy is approximated as the product of solute size with the square of solvent solubility parameter $(\delta)$, $=1.22 \Delta G^{\circ} \mathrm{CH}_{2}$ is expected to correlated linearly with $\delta$. The solubility parameter is defined as the square root of the solvent heat of vaporization per unit solvent volume $(\mathrm{mL})$.

$$
\Delta G_{\mathrm{CH} 2}^{\circ}=h_{1}+h_{2} \delta^{2}
$$

In equation $8, k_{1}$ and $k_{2}$ are regression coefficients for the given șistem

\section{Results and Discussion}

First of all all the slopes in the plots of $\ln K \mathrm{w}$ solute carbon number were found positive. which means that the free energy of transfer of $\mathrm{CH}_{2}$ unit from the gas phase to a solvent is negative (favorable) for all the solvents and that the absolute magnitude of interaction free energy is always larger than the absolute magnitude of cavity formation free energy (See equation 7). However. the slope change is dependent only on the cavity formation free energy since the interaction free energy is virtually invariant while the cavity formation free energy is not. The plots are shown for typical nonpolar and polar solvents in Figure 1. The slope gets lower as the solvent polarity gets higher. The cavity formation

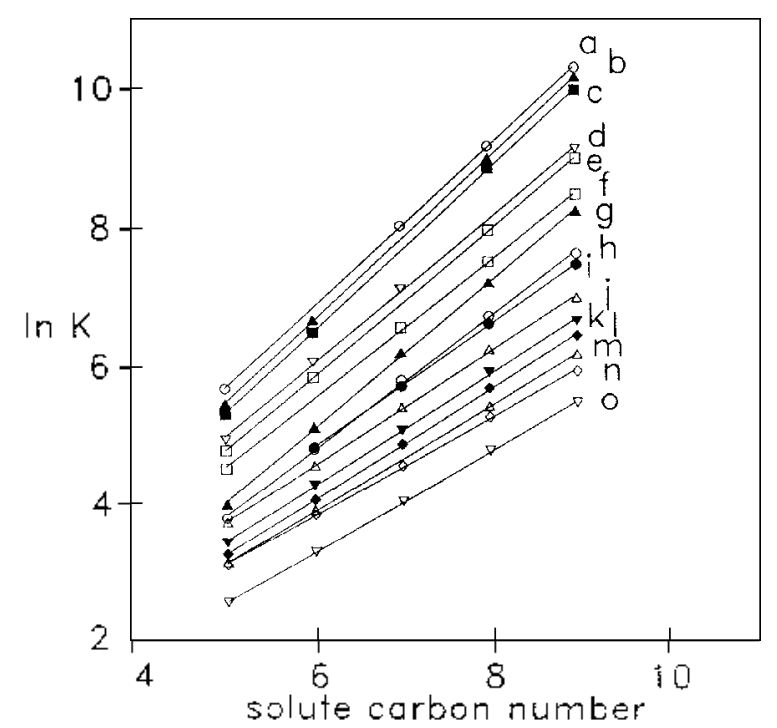

Figure 1. The plot of $\ln K$ is. solute carbon number for some selected solvents. a: hexane, $b$ : tetrahydrofuran, $c$ : benzene, $d$ : dichloroethane, e: ethylacetate, f: acetone, g: hexamethylphosphotriamide, h: dimethylacetamide, i: methanol, i: acetonitrile, $\mathrm{k}$ : perfluorooctane, l: N-methylforamide, $\mathrm{m}$ : nitromethane, $\mathrm{n}$ : trifluoroethanol, 0 : dimethy sulfoxide.

energy is always positive and gets larger as the solvent polarity gets higher. The interaction energy is alway's negative and serves as a dominant factor in the transfer free energy. Thus the slope is positive owing to the interaction free energy and is lowered by the increase of opposing cavity formation energy, in other words. the slope decreases with the increase of solvent polarity as shown in Figure 1.

We calculated the methylene group transfer free energy for each solvent from its slope in the plot of $\ln K$ us. solute carbon number and plotted it against the solvent colnesive energy density (square of solvent solubility parameter. $\delta$ ) in Figure 2 to examine if $\delta^{2}$ is a good measure of $\mathrm{CH}_{2}$ cavity formation free energy. As can be seen in Figure 2, the correlation is not good $(r=0.78 . n=45)$ although a coarse correlation is observed. The cavity formation is a rather

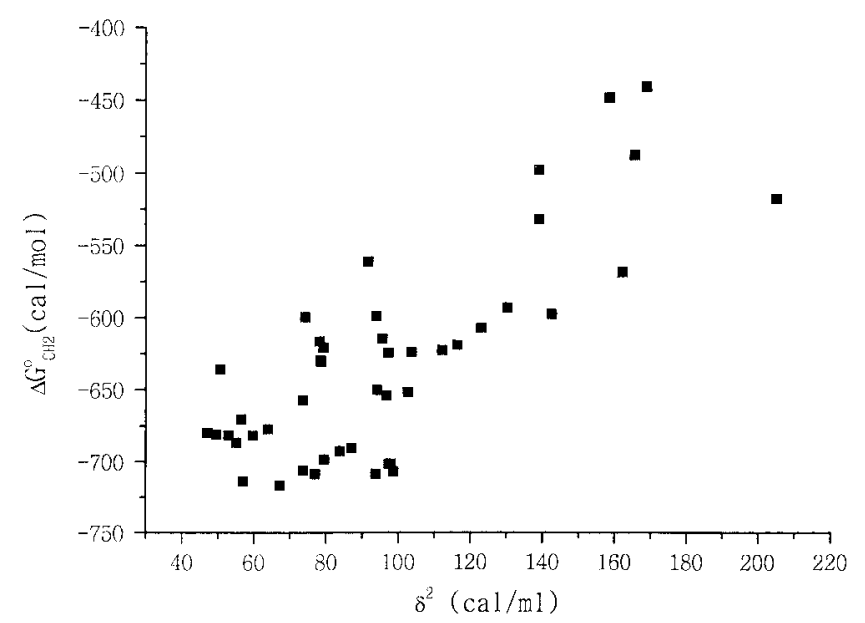

Figure 2. The plot of transfer free energy of methylene group vis. solvent cohesive energy density $\left(\delta^{2}\right)$. 


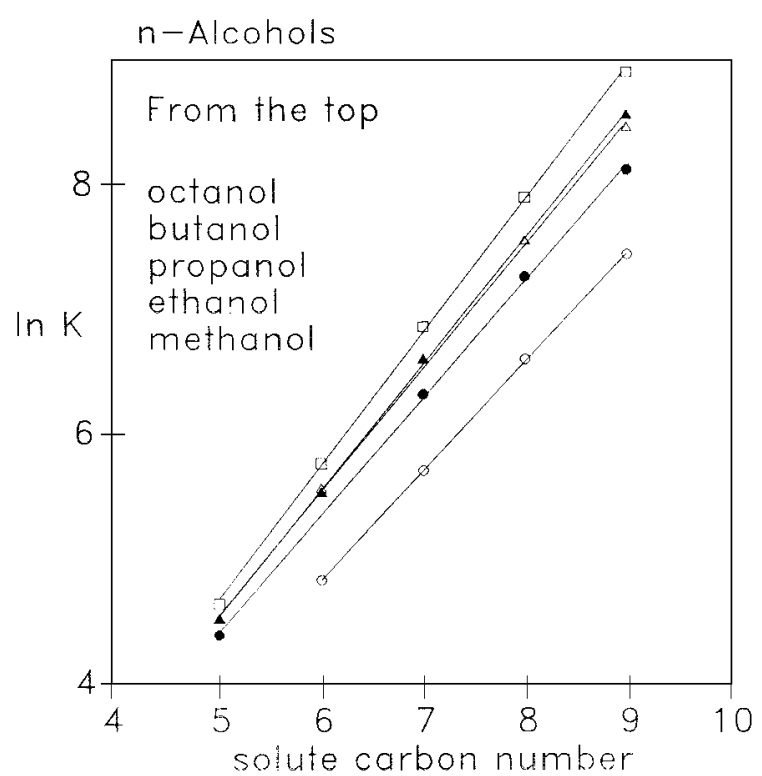

Figure 3. The plot of $\ln K$ is. solute carbon number for $n$-alcohols.

complicated process. and its free energy change may not be described by a single major parameter.

We examined the change of slopes for a couple of homologous series of solvents. alcohols (Figure 3) and nitriles (Figure 4). The slopes of larger members of the series are higher than those of smaller members. thus the order(from the highest) is octanol. butanol. propanol. ethanol. methanol for the alcohols. and pentadecanitrile. nonanenitrile butyronitrile acetonitrile for the nitriles. We again see that the slope decreases with the increase of solvent polarity in Figures 3 and 4 .

Next. we plotted $\ln K$ against solvent carbon number for three homologous solvent series. alkanes. alcohols. and nitriles to see how the gradual solvent structure and polarity change affects the free energy of solute transfer. The plots are shown in Figures 5 (alkane solvents). 6 (alcohol solvents).

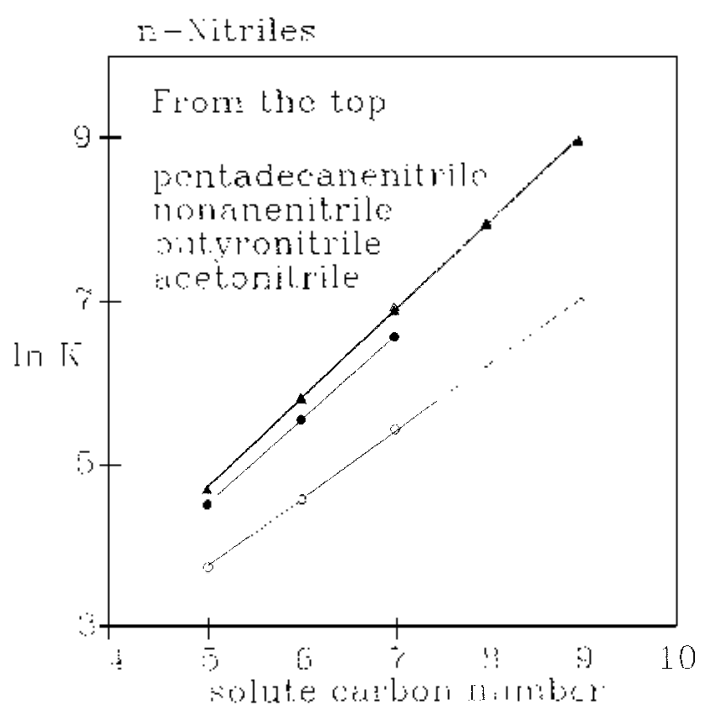

Figure 4. The plot of $\ln K$ vs. solute carbon number for $n$-nitriles.

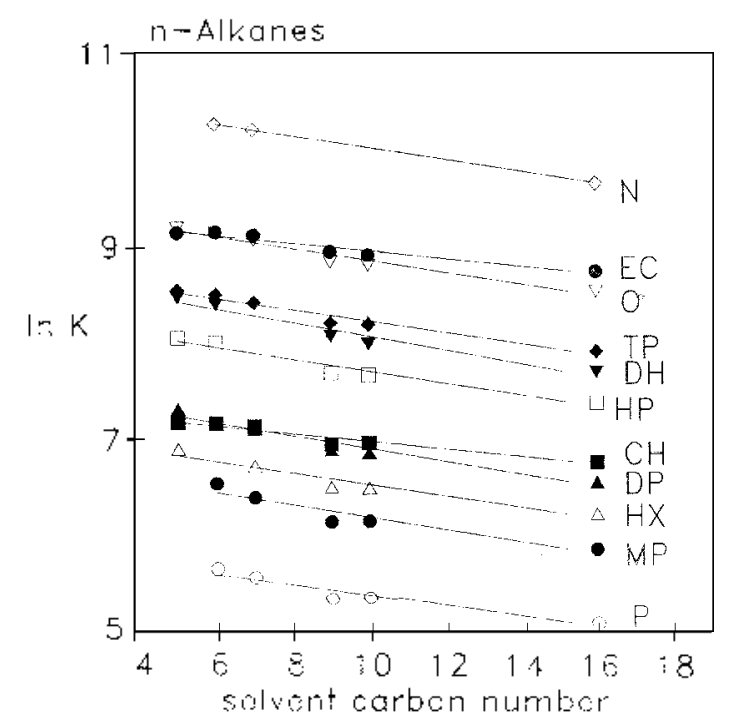

Figure 5. The plot of $\ln K$ is. solvent carbon number of $n$-alkanes $<$ Solute abbreviation> P: $n$-pentane, HX: $n$-hexane, HP: $n$-heptane, O: octane, $N$ : nonane, MP: 2-methylpentane. DP: 2,4-dimethylpentane, CH: cyclohexane, TP: 2,3,4-trimethylpentane, EC: ethyloyclohexane.

and 7 (nitrile solvents). The individual solvent groups show characteristic variation trends with respect to solvent carbon number. The value of $\ln K$ decreases with solvent carbon number in the plot of alkanes (Figure 5). However. it increases abruptly and levels off later in the plot of alcohols (Figure 6), while it increases abruptly, levels off, and finally decreases a little bit in the plot of nitriles (Figure 7).

An increase in $\ln K$ corresponds to a decrease in cavity formation energy or to an enhanced solute-solvent interaction (more negative interaction energy'). Thus, the linear decrease of $\ln K$ with solvent carbon number in alkane solvents (Figure 5) means either an increase of cavity formation energy or decreased solute-solvent interaction with an increase of solvent carbon number. A decrease of solutesolvent interaction with increase of solvent carbon number is unlikely, while an increase of cavity formation energy with solvent carbon number is consistent with the trend of solvent solubility parameter. ${ }^{21.22}$ Higher alkanes have larger solubility parameters. A decrease of cavity formation energy (decrease in $\ln K$ ) with decrease of solute size (from nonane to pentane) is surely observed. too in Figures 5, 6. and 7.

An increase of $\ln K$ with solvent carbon number of alcohols (Figure 6) is also owing to cavity formation energy: As the number of $\mathrm{CH}_{2}$ units of alcohol solvents increases. the solvent structure becomes less polar. and its solubility parameter and the cavity formation energy decrease $(\ln K$ increases). The solvent polarity decrease is rather high when the solvent is changed from methanol to ethanol. but the difference gets lower and lower as the solvent is changed to larger alcohols (propanol, butanol, octanol).

An increase of $\ln K$ with solvent carbon number of nitriles (Figure 7) can also be explained in terms of cavity formation energy as in the case of alcohol solvents (Figure 6). The $\ln K$ value of Figure 7 (nitrile solvents) approaches to a plateau 


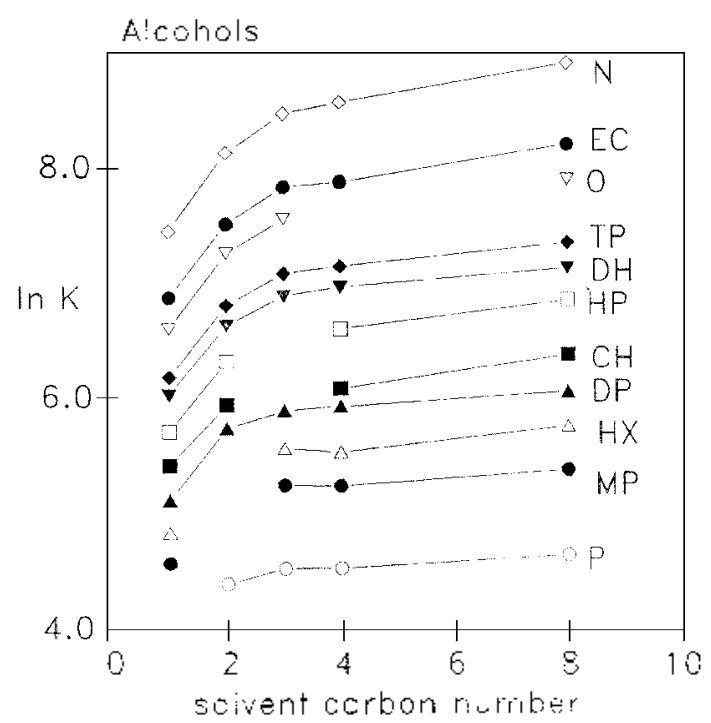

Figure 6. The plot of $\ln K v$ s. solvent carbon number of $n$-alcohols. $<$ Solute abbreviation> P: n-pentane, $\mathrm{HX}$ : n-hexane, HP: n-heptane, O: octane, N: nonane, MP: 2-1nethy lpentane. DP: 2,4-dinethylpentane, $\mathrm{CH}$ : cyclohesane, TP: 2,3,4-trimethylpentane, EC: ethyloyclohexane.

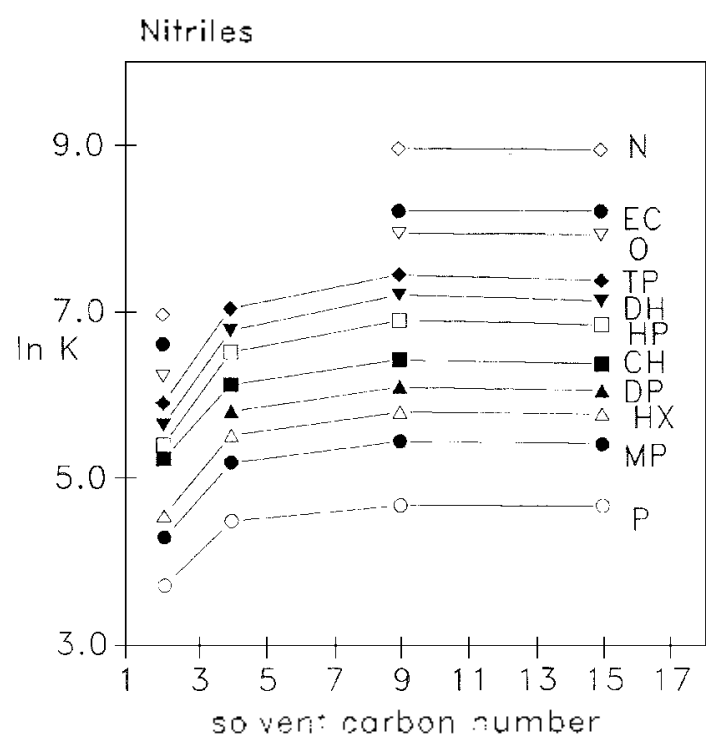

Figure 7. The plot of ' $K$ n vs. solvent carbon number of $n$-nitriles. $<$ Solute abbreviation> P: n-pentane, HX: n-hexane, HP: $n$-heptane, O: octane, N: nonane, MP: 2-methylpentane. DP: 2,4-dinethylpentane, $\mathrm{CH}$ : cyclohexane, TP: 2,3,4-trimethylpentane, EC: ethylcrclohexane.

much faster than that of Figure 6 (alcohol solvents). This phenomenon is consistent with the fact that nitriles are generally less polar than alcohols. An interesting observation in Figure 7 is slight decrease of $\ln K$ when the solvent is changed from nonanenitrile to pentadecanenitrile. A decrease of $\ln K$ (an increase of cavity formation energy) with solvent carbon number is a characteristic feature of alkane solvent group as shown in Figure 5. Thus, even solvents with a polar functional group can be regarded as virtually nonpolar solutes if the number of $\mathrm{CH}_{2}$ units gets large enough.

\section{Conclusion}

We examined the effect of solvent structure on the free energy of transfer of a $\mathrm{CH}_{2}$ unit from the gas phase into a solvent by comparing the slopes in the plots of $\ln K \psi$ solute carbon number among various solvents. The slope in the plot of $\ln K$ ws. solute carbon number was alway's positive and its value decreases with increase of solvent polarity. Especially: we clearly observed a gradual slope increase with increase of solvent carbon number for a series of homologous solvents. We observed characteristic trends in the plots of $\ln K$ w. solvent carbon number for individual solvent groups. Such peculiar trends were related to variation in cavity formation energy. When a structural change of a polar solvent is made by sequential addition of $\mathrm{CH}_{2}$ units. an abrupt polarity decrease. then gradual levelling off. and finally, conversion to a virtually nonpolar solvent take place.

Acknowledgement. This work was the result of the 2002 project of Center for Advanced Bioseparation Technology at Inha University supported by Korea Science and Engineering Foundation.

\section{References}

1. Ashworth, A. J. J. Chent. Soc. Faraday II 1973. 69. 459.

2. Ashworth, A. J.; Everett. D. H. Trais. Faraday Soc. 1960. 56 , 1609.

3. Cruickshank. A. J. B.: Windsor. M. L.: Young. C. L. Proc. Ron? Soc. A 1966. 295.271.

4. Conder. J. R: Purnell. J. H. Trans, Foradoy Soc. 1963, 59. 1655 .

5. Martire. D. E.; Pescsok, R. L.: Purnell, J. H. Trans. Faraday Soc. 1965.61 .2496 .

6. Abraham. A. H.: Liszi. J. J. Chem. Soc. Foraday Soc. 1978. 74. 16014.

7. Abraham. M. H. J. Am. Chem. Soc. 1979. 101, 5477.

8. Prausnitz, J. M.; Lichtenthaler. R. M.: de Azevedo, E. G. Molecular Thermodnamics of Fluid Phase Equilibria. 2nd Ed. Prentice Hall: Englewood Clitts. 1986.

9. Walas. S. M. Phase Equilibria in Chemical Engineering. Butterworth Publishers: Boston. 1985.

10. Castells, C. B.; Carr, P. W.: Eikens, D. I.: Bush. D.: Eckert, C. A. Ind Eng. Chent Res 1999, 38. 4104.

11. Conder. J. R.: Young, C. L. Phusicochemical Measwmements by Gas Chomatography: Wiley-Interscience: New York. 1978.

12. Cheong. W. T. Bull. Konan Chem. Soc. 2002. 23. 459

13. Kovats. E. Ad Chrontatogr: 1965. 1.229.

14. Hildebrand, J. H.: Scott. R. L. Regular Solutions: Prentice-Hall: Englewood Clitt; New Jersey, 1962.

15. Scatchard, G. Chem. Rev $1931,8,321$.

16. Wilianson. A. G. Am. Rev Phus. Chem. 1964. 15.63.

17. Cruichshank. A. J. B.: Windsor. M. L.: Young. C. L. Proc. Ro? Soc. A 1966. 295.271.

18. Guggerheim. E. A. Proc. Rov Soc. A 1944, 183. 213.

19. MeGlashan. M. L: Morcom. K. W. Williamson, A. G. Trams. Faraday Soc. 1961. 59,601.

20. Luckhurst. G. R.: Martire. D. E. Trons. Farado Soc. 1969. 65. 1248 .

21. Hildebrand. J. H: Prausnitz. .T. M.: Scott. R. L. Regutor and Related Solutions; Van Nostrand: New York, 1970.

22. Shidoda. K. Principles of Solution and Solubility Marcel Dekker: New York, 1978. 